\title{
The Quantum Computing Business Ecosystem and Firm Strategies
}

\author{
Joseph Jenkins \\ University of Minnesota \\ jenki627@umn.edu
}

\author{
Nicholas Berente \\ University of Notre Dame \\ nberente@nd.edu
}

\author{
Corey Angst \\ University of Notre Dame \\ cangst@nd.edu
}

\begin{abstract}
Quantum computing is an emerging technology that promises to revolutionize business and society. Although it is still in its early stages, firms have begun to invest heavily in the technology. In this article, we review some key themes of quantum computing from a business-oriented perspective, and construct a framework of the quantum computing business ecosystem. We also conduct an analysis of the contemporary discourse to identify four general strategies that firms are following as they invest in quantum computing. We refer to these as conventional, options, discovery, and adversarial strategies and describe and offer examples of each.
\end{abstract}

\section{Introduction}

Quantum computing is rapidly becoming a reality, and the possibilities for dramatic disruption to traditional computing are seemingly endless. Quantum computing is expected to dramatically reshape society vis-à-vis the advantages it offers to industries such as medicine, finance, transportation, and energy [1-4]. So, it should not be surprising that many firms are investing heavily in quantum. A recent survey of Fortune 1000 decision-makers found that $81 \%$ have quantum use cases in mind, and $61 \%$ believe the technology will significantly impact their industry [5].

An abundance of research exists on the technical and scientific aspects of quantum computing, as well as into the sorts of problems that quantum can solve [6-8]. But there is, as yet, little research exploring how firms are approaching this technology from a strategic perspective, which is a significant omission that should not be overlooked. Quantum computing represents an order of magnitude performance improvement over previous generations of computing, and quantum-powered artificial intelligence (AI) is likely to impact virtually every aspect of human existence. Quantum computing represents a tremendous opportunity, and we contend that firms should incorporate attention to it in their strategic decision-making. To do so, they need to understand the quantum computing business ecosystem as the context for this revolution in computing and the approaches other firms are taking to address the opportunity. Therefore, we ask: What is the business ecosystem for quantum computing? What are strategies that firms are taking with respect to quantum computing?

To address these questions, we conduct a focused review of scholarly and practitioner literature on business applications of quantum computing. From this review, we make two contributions. First, we introduce and characterize the contemporary business ecosystem for quantum computing. This ecosystem is composed of hardware, infrastructure, delivery, quantum business strategies, and applications, all of which we describe later in the paper. Second, we identify four general strategic approaches that firms currently take in their investments in quantum computing. These are:

- Conventional strategy: firms use quantum computing to solve common computing problems with dramatically improved performance.

- Options strategy: firms are investing in experimental pilot projects to explore potential future applications and build capabilities.

- Discovery strategy: firms are using quantum computing to address previously impossible problems.

- Adversarial strategy: firms are using quantum computing for contexts that involve dynamic, real-time arms race competition.

The observations provided in this study expand on previous articles that provided an overarching view of the commercial landscape to develop an ecosystem and strategies [7-9]. Below, we provide a brief overview of the underlying technology, explore the ecosystem surrounding quantum computing, and identify and describe four strategic approaches.

\section{Background}

At the heart of quantum computers is quantum physics. In the 1980s, scientists began to speculate that they could manipulate the laws of quantum physics to speed up computers [10]. Successes in quantum 
computing arrived in force with the new millennium. For example, D-Wave's quantum annealer that arrived in 2007 achieved strong performance on certain optimization and sampling problems [11]. Since then, companies have been racing to build universal quantum computers, find use cases, and develop relevant applications. Next, we briefly sketch the very basics of quantum computers. For more detailed information, other sources are readily available (e.g., $[7,12,13])$.

The power of quantum computers originates from technology operating via qubits as opposed to bits. Traditional computers are composed of bits, which can hold a value of 1 or 0 . Quantum computers work using qubits, which can store values of 0,1 , or exist in a superposition where the qubit holds both values simultaneously $[13,14]$. Eventually, when the quantum computer measures the state, the system collapses, and the qubit has a value of 1 or 0 . The existence of superposition allows for entanglement, which provides that multiple qubits interact and hold all values simultaneously [12]. As a result of utilizing superposition and entanglement, quantum computers exponentially improve performance for certain types
Institute for Quantum Information and Matter, compared quantum computing to reading a 100-page book [12]. For each page you read, you learn another $1 \%$ of the content in the book (traditional computing) [12]. Whereas, quantum computing analyzes the content of the book collectively at once [12]. We are now entering the era of what some refer to as the Noisy Intermediate-Scale Quantum (NISQ) phase, where quantum is just beginning to realize its potential, and with it, experiments will be conducted to identify its business applications and prepare for advances in future hardware [17].

Recent developments in quantum computing have demonstrated a few classes of problems the technology appears suited to address. Konstantinos Karagiannis, head of quantum computing services at Protiviti and host of Post-Quantum World podcast, along with IBM, identified optimization, machine learning, and simulation as three areas quantum computing will excel $[18,19]$. Optimization appears to be the area that offers the most near term promise. Businesses have relied on optimization for quite some time; however, quantum computing will provide better approximate solutions (and in some use cases, the

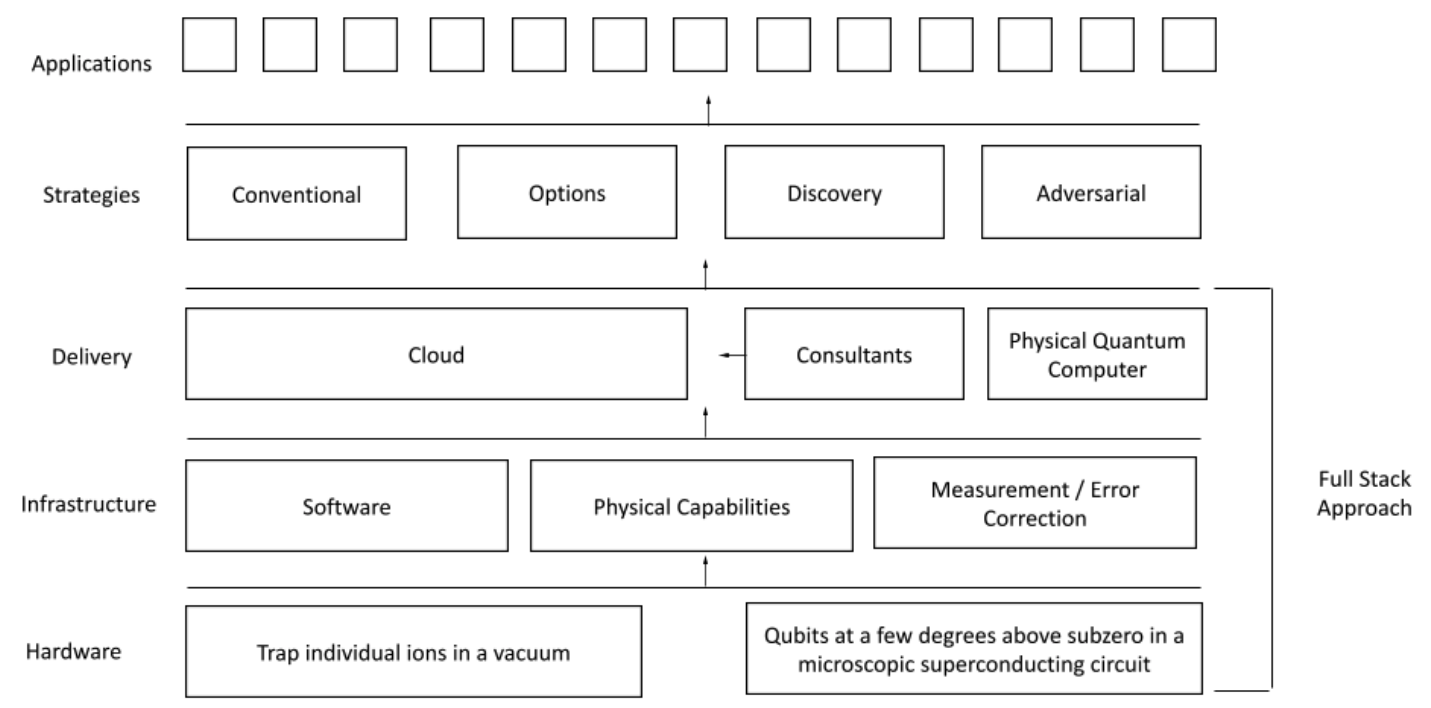

Figure 1: Quantum Computing Business Ecosystem

of problems. An n-qubit quantum computer can process $2^{\mathrm{n}}$ values in parallel $[15,16]$. Two qubits in superposition and entanglement can hold up to four values based on all possible values of 1 and 0: 00, 01, 11 , or 10. Three qubits in superposition and entanglement hold up to eight values: 000, 001, 010, $100,110,011,101$, and 111. Thus, a 20-qubit quantum computer will hold over one million values in parallel. John Preskill, Cal Tech's director of the exact solution) over classical computers [12]. Most scholars and industry experts seem to agree that in the near term, a hybrid quantum-classical algorithm will be the most viable optimization solution [19-22]. Quantum computers are also suited for quantum simulation with use cases in chemistry, pharmaceuticals, and material science product design.

Another promising near-term application is quantum simulation. Loosely, it is defined as 
"controllable quantum systems that can be used to simulate other quantum systems" [23]. Presently, there are two dominant methods: analog quantum simulators and digital quantum simulators. Analog quantum simulators refer to a system with qubits that exhibit a dynamic similar to the system represented and under study [24]. Meanwhile, a digital quantum simulator is a gate-based system that, when programmed correctly, can simulate any physical system [24]. Regardless of the approach, quantum computers hold a significant advantage over classical computers in quantum simulation due to the fact that quantum computers naturally exist as a quantum system, and thus, they store large amounts of information in a small space. This opens the door to testing various models and situations that currently pose challenges or remain unsolvable using classical computers [25]. Use cases, such as simulating chemical interactions, will revolutionize the pharmaceutical and chemical industry by expediting the research and development time frame and reducing expenses [23]. Society will benefit immensely from rapidly identifying potential solutions to treat disease and health conditions without running an actual lab experiment. The potential for simulation applies to a host of other industries, as well. For example, automakers are exploring the feasibility of the technology to simulate heat and mass transfers, fluid dynamics, and compressible flows [26]. The longerterm benefit of quantum simulation allows companies to digitally recreate highly complex interactions, which will enable them to develop products cheaper, better, and faster than previously possible.

Quantum computing offers promising competitive advantages to early adopters due to potential insights from big data and deep learning. Experts foresee quantum machine learning to speed up computationally intensive algorithms, including machine learning, clustering, classification, and pattern matching [27]. The challenge facing quantum lies with identifying problems the technology currently can address and solve faster than traditional computers. As hardware and infrastructure become more advanced, the complexity and number of applications will grow.

\section{Quantum Computing Business Ecosystem}

In 2012, John Preskill coined the phrase "quantum supremacy ${ }^{1}$ " to describe the moment when quantum

\footnotetext{
${ }^{1}$ Opinions differ with regard to whether 'quantum supremacy' and 'quantum advantage' are distinct phrases with subtly different meanings. Some think of quantum
}

computers solve tasks and problems that cannot be realistically solved by conventional computers [17]. $\mathrm{He}$ claimed that 30 years is "not an unrealistic timescale" for general-purpose quantum computing supremacy [29]. However, today, some areas show promise of reaching quantum supremacy soon, while others are still in their infancy. For example, in 2019, Google claimed their Sycamore quantum processor solved a problem in three minutes that would take a conventional computer over a thousand years [30]. Nevertheless, the space parallels the development of early conventional computers [7]. Today, companies in the quantum landscape vary from hardware-focused startups to large technology companies delivering quantum computing via the cloud and established businesses exploring its applications. We dub this space the "quantum computing business ecosystem," summarized in Figure 1, composed of hardware and infrastructure, delivery, strategies, and applications.

\subsection{Hardware \& Infrastructure}

At the forefront of the industry is the hardware and infrastructure driving the entire quantum domain. The development of quantum hardware has followed or exceeded Moore's Law year over year, providing optimism the hardware will significantly impact select applications and use cases [20, 31, 32]. Presently, IBM has a 65 qubits general-purpose quantum computer but aims to release a 127 -qubit quantum computer by the end of the year in the hopes of reaching over 1,000 qubits by 2023 [33, 34]. The various companies involved in the ecosystem's hardware and infrastructure portion elected to prioritize distinct objectives. For instance, Google's approach aims to create the best infrastructure, IBM wants to deliver the entire enterprise through their IBM Q Experience, and Microsoft is committed to focusing on developers by releasing the Microsoft Quantum Development Kit [22]. The delivery and hardware space of the quantum business ecosystem is exciting and collaborative, with many startups and large tech companies working together to push the technology forward.

One aspect of quantum computing that resides within the hardware is the annealing process. Quantum annealing uses quantum physics to find low-energy states of a problem and capitalize on them. This hardware is already commercially available and easy to scale, which is why most of the problems quantum computers address are optimization-based [15, 35]. In the quantum stack, the strategies firms are exploring

advantage as incremental improvements from quantum over conventional. For the purpose of our paper, we follow Preskill (2019) and consider them to be equivalent. [28] 
can be broken down into conventional, options, discovery, and adversarial (we discuss these in detail in section 3.3). Finally, the last layer of the ecosystem is a firm's applications of the technology. Some of these are being tested internally, some externally, and others are merely thought-projects, but excitement and focus in the business community are emerging. Quantum computing, like most emerging technologies, will provide competitive advantages for early adopters, which is why many companies are beginning to explore the technology today.

Developing quantum hardware and infrastructure is one of the most formidable challenges the quantum business ecosystem faces to turn this technology into an everyday business reality. The hardware will have to overcome quantum error correction, which protects quantum systems by encoding information into a highly entangled state [12]. The task naturally becomes more difficult as the number of qubits increases. Thus far, two dominant approaches to create highly entangled states have emerged. The first trap individual ions into a vacuum by utilizing electric and magnetic field properties [36]. The second operates at a few degrees above absolute zero and integrates qubits with superconducting circuits [36]. The companies leading the charge include Cambridge Quantum Computing, D-Wave, Honeywell, IBM, and Regetti, to name a few.

However, quantum hardware will likely not entirely replace conventional computers, certainly not all at once, if ever. Instead, developments in the ecosystem will come in waves. The consensus appears to be that we will go through three generations of quantum computers: the noisy intermediate-scale quantum (NISQ) era, broad quantum advantage, and full-scale fault tolerance $[12,37]$. The first generation of quantum computers, expected to last until 2028, will find use cases for the technology in simulations, optimization problems, and continued exploration of other suitable applications [19, 38]. The second generation of quantum computers will achieve quantum supremacy in a wide variety of applications and will overcome hurdles currently present in error correction, making the technology more reliable [37, 38]. Finally, by 2035 quantum computers should reach full-scale fault tolerance, enabling them to tackle some of the most complex problems in society and widespread commercial adoption. The expected timeframe projects limited general application use in the immediate future, but niche-specific quantum computers will likely make a significant impact soon.

\subsection{Delivery}

As the Quantum Business Ecosystem continues to develop, it appears that the vast majority of companies will access quantum computing via the cloud. A recent International Data Corporation (IDC) survey of executives found that $65 \%$ of respondents expect to access quantum computing via the cloud [25]. Two primary approaches to quantum computing have emerged: the full-stack approach (IBM) and the partial stack approach (Microsoft and Amazon). The fullstack approach develops hardware and infrastructure built by the company and accessed by end-users through the cloud, while the structural open innovation approach centers around investing in various qubit architectures to provide cloud solutions [8]. Regardless of the approach companies elect to take, one thing is certain, the Quantum Business Ecosystem will center on the cloud.

Yet, delivery in the ecosystem is not limited to cloud-based providers; consulting companies including Accenture, Boston Consulting Group (BCG), Deloitte, McKinsey \& Company, and Protiviti have set up divisions and emerged as trusted voices advising companies on the near-term applications of quantum. The industry's challenge is the software requirements and new programming languages software engineers will need to learn $[20,39]$. Tech companies are developing languages, including Q\#, Qiskit, and Cirq, based on the Python programming language to get programmers started in familiar environments [14]. The importance of fostering a community of developers should not be overlooked in the ecosystem, as there is much room to grow in software development, compilers, and making learning materials easily accessible [39, 40]. The newfound capabilities and unexplored possibilities of quantum computing require that all levels of the ecosystem interact to form a consensus the exploration of the technologies' capabilities continue to be explored.

\subsection{Quantum Strategies}

IBM identified five steps firms progress through as they develop a quantum strategy: selecting champions, identifying use cases, experiment, chart your course, and flexibly adapt [19]. Each of these tactical steps build off knowledge gleaned from prior steps [19]. While this approach is useful for IBM and other firms wishing to capitalize on quantum computing, it does not provide an over-arching landscape of strategies for services and applications offered by quantum, which is the aim of our investigation. 
We conducted a focused literature search on academic and practitioner research, as well as in the broader media. We discovered that firms across many industries are beginning to explore quantum computing for select applications to determine what can be used and implemented in the near term. Our review produced four general strategies in the quantum ecosystem: conventional, options, discovery, and adversarial strategies. These strategies are not necessarily mutually exclusive, in that the same firm could be pursuing more than one strategy, but collectively they are exhaustive for the contemporary discourse.

We began our search by looking for academic articles that investigated the general capabilities of quantum computing, applications, and projected use cases. The technical and scientific literature on quantum computing is dense and abundant, but literature on applications in business is notably sparse. Through search terms and snowball sampling, we identified 35 scholarly articles that addressed the business applications of quantum computing. Next, we searched practitioner-focused articles and reports by searching the websites of technology companies, technology consultants, management consultants, and strategy consultants for white papers and technical reports. This search yielded 74 articles. Finally, we used Factiva from Dow Jones to review broad media, including press releases, articles from sources including the Financial Times and Wall Street Journal, and general company news, which yielded 106 articles. Selection criteria for articles included in our sample were (1) explicitly mentioned quantum computing; (2) described a business use case.
We coded our research dataset in three general rounds. Coding was iterative and collaborative among the authors, but the first coding round involved finding the general classes of organizational use cases. Use cases identified from the scholarly articles were exhaustive. We organized these in a variety of ways including by topic and application. In the second round of coding, we identified organizations investing in quantum computing in various manners. Finally, we identified the thematic codes of the general strategies that we identified. Overall, this dataset and our approach to coding quantum computing strategies effectively reflect the contemporary public discourse. We identified four general strategies - what we named: conventional, options, discovery, and adversarial strategies (see Table 1). Next, we address each.

Conventional Strategy: The conventional strategy for using quantum computing lies in the technology's ability to perform specific optimizations and simulations with incredible performance improvements. This strategy essentially involves solving problems that firms currently use traditional computing, but they will do it much faster with quantum.

The traveling salesperson problem was long ago established as a computationally-intensive problem. A similar problem arises when considering shipping logistics, traffic optimization, or scheduling delivery trucks in an urban environment. There are over $2^{\wedge} 1,000,000$ combinations of shipping routes, which makes the problem infeasible on anything other than a quantum computer [41]. ExxonMobil partnered with

\begin{tabular}{|c|c|c|}
\hline Quantum Strategy & Approach to Quantum Computing & Examples \\
\hline Conventional & $\begin{array}{l}\text { Traditional problem solving with far } \\
\text { superior performance. Optimize paths } \\
\text { through a problem space. }\end{array}$ & $\begin{array}{l}\text { - Ford and NASA optimizing driving routes to } \\
\text { reduce energy consumption } \\
\text { - Port of Los Angeles optimize of containers }\end{array}$ \\
\hline Options & $\begin{array}{l}\text { Build capabilities through pilot projects. } \\
\text { Focus on the potential for the firm to learn } \\
\text { and capitalize on quantum in the future. }\end{array}$ & $\begin{array}{l}\text { - Save-On-Foods are developing quantum } \\
\text { algorithms } \\
\text { Qubit Engineering is placing windmills for } \\
\text { maximum energy production }\end{array}$ \\
\hline Discovery & $\begin{array}{l}\text { Open up new approaches to inquiry in } \\
\text { ways that were previously impossible. } \\
\text { Explore very large problem spaces. }\end{array}$ & $\begin{array}{l}\text { - OTI Lumionics computational chemistry } \\
\text { simulations of new materials } \\
\text { - IBM and Mitsubishi molecular structures of new } \\
\text { efficient OLED materials } \\
\text { - NASA and Google identify new materials to be } \\
\text { used for space travel }\end{array}$ \\
\hline Adversarial & $\begin{array}{l}\text { Real-time competitive decision making. } \\
\text { Looking to succeed in contexts with "arms } \\
\text { race" dynamics. }\end{array}$ & $\begin{array}{l}\text { - BBVA optimize the trading path for a 52-asset } \\
\text { investment portfolio } \\
\text { - Accenture develop algorithms for currency } \\
\text { arbitrage, and trading }\end{array}$ \\
\hline \multicolumn{3}{|c|}{ Table 1. Generic Quantum Business Strategies } \\
\hline
\end{tabular}


IBM to investigate applications in maritime shipping, such as capacity limitations, optimal routes, port locations, and time windows, to increase efficiencies [42-44]. The use of quantum in transportation also extends to optimizing travel for cars, public transit, taxis, and ride-sharing. In 2016, Volkswagen used quantum computing to optimize the airport routes of 10,000 taxis in Beijing and created applications that optimize public transportation routing in seconds that took a conventional computer 45 minutes [7, 32]. They also tested their application in 2019 in Lisbon to coordinate the city's buses during the Web Summit technology conference [7, 45]. Most scholars and industry experts seem to agree that in the near term, a hybrid quantum-classical algorithm to solve optimization problems will be the most viable solution [23, 24, 31, 46]. Volkswagen has also explored a new traffic management service using a hybrid approach by analyzing movement data using traditional computers and optimizing taxi, public transportation, or ridesharing placements via quantum computers based on the determined demand spots and different destinations [47, 48]. Similarly, Ford and Microsoft explored using Bing Maps and a hybrid quantumclassical algorithm that delivered results in 20 seconds [49]. In small test markets with 5,000 vehicles, the application reduced total congestion by 73 percent compared to "selfish" driving, improved commute time by 8 percent, and saved drivers 55,000 hours otherwise spent in traffic [49, 50]. The space and strategy in routing and logistical management have room to grow and call for further experiments, but all signs point that quantum computers will significantly impact the industry.

Options Strategy: The most frequent trend we observed involved organizations exploring quantum technology and building capabilities. Much quantum computing is still emerging, but companies are positioning themselves by developing algorithms and applications once the hardware reaches a commercially viable everyday use threshold. Organizations including Daimler, AG, Samsung, JSR Corporation, Hitachi Metals, Oak Ridge National Lab, and Oxford University partnered with IBM for this very reason [51]. Companies are taking the optionsbased approach we still do not know what tasks quantum computers will achieve "quantum supremacy" over conventional computers. We noticed companies placing bets in various quantum applications, hardware, and processes so they are not left on the outside when the technology matures.

Deep learning has become one of the hottest topics in the past twenty years as the technology has significantly impacted science, business, and broader society. Quantum machine learning promises to speed up computationally intense algorithms, including machine learning, clustering, classification, and pattern matching [7]. One notable characteristic of quantum deep learning is that it does not require a general-purpose quantum computer but rather quantum annealers, which are easier to build and scale [30]. Quantum deep learning can perform supervised and unsupervised machine learning; however, like many things in quantum space, we do not know which areas will provide advantages over conventional computing [24]. One challenge facing quantum deep learning is the time it takes to load and unload data. Before running a quantum algorithm, the input data needs to be encoded for quantum, and once the algorithm finishes, the result itself is in a quantum state that needs to be measured [24, 30]. The fact remains that there is much about quantum deep learning that is still evolving, which is why many companies are identifying and experimenting with potential applications. Volkswagen, BMW, and Google have used D-Wave quantum computers to train algorithms with large amounts of driving data in their quest to build self-driving cars [52]. Scientists explored using quantum deep learning to compute Google PageRanks [53]. Monte Carlo machine learning algorithms align well for quantum deep learning, which Goldman Sachs and QC wire began exploring to run partial simulations that beat conventional computing [33, 42]. While scientists and business leaders continue to search and experiment with quantum deep learning applications, as hardware and knowledge continue to improve, machine learning will become more frequently run on quantum machines.

Boeing Co. teamed up with AWS to explore the technologies capabilities to speed up materials-science research, while Lockheed Martin used D-Wave computers to test whether a piece of software is bugfree, which they claim is impossible to do on classical computers [7, 11, 46]. Some of the most considerable advances quantum computers will provide to the greater society will be in healthcare. The industry is still building its capabilities, but companies like Siemens are exploring the technology's ability to perform faster image detection to identify unhealthy patients from healthy patients $[50,54,55]$. Yet, the same characteristics that make quantum computers so promising present its biggest threat: the potential to break encryption. IBM recently announced cloud solutions that they believe will be quantum-safe cryptography, and financial companies, including JPMorgan and Visa, are conducting internal research [56-59]. The overarching goal for many companies is to build quantum capabilities and options around this 
emerging technology. As Andrew Fursman, CEO at 1Qbit stated: "maybe a million-dollar investment in order to understand something that could jeopardize your multibillion-dollar business is a great trade-off at this stage" [53].

Discovery Strategy: Another strategy that appears consistently across the ecosystem is development discovery. We define development discovery as the process of undergoing research and development in the hopes of identifying and navigating a problem space of unprecedented scope. Quantum computers' ability to hold multiple values simultaneously lends itself towards simulation and may significantly reduce development time and expenses [21]. For example, companies across sectors are investigating and researching the application of quantum computers to simulate chemical reactions. Mercedes Benz, Mitsubishi Chemical, and Volkswagen are utilizing quantum computers to simulate chemical reactions in hopes of creating better batteries [24, 60, 61]. Mercedes intends for half of its car sales to be plug-in hybrids or all-electric vehicles in ten years, and they view quantum computers as one of the tools that will aid them in succeeding in this goal [61]. In the energy space, IBM has partnered to explore the technology's capabilities to discover new clean energy sources, electric grid management, and modeling environmental and climate change $[62,63]$. In the healthcare space, Menten AI used hybrid quantum programs to identify de novo protein designs that outperformed traditional computers, and they used them to test against the COVID-19 virus [64]. Yet, the technology also offers the opportunity for significant discoveries in big data and machine learning [52]. The Cleveland Clinic recently announced a ten-year partnership with IBM to explore the technology's applications in areas including "genomics, single-cell transcriptomics, population health, clinical applications, and chemical and drug discovery" [65]. In 2018, Ford and NASA's Quantum Artificial Intelligence Laboratory (QuAIL) teamed up to explore autonomous vehicle research [66, 67]. Ken Washington, Ford's chief technology officer, said, "for us, quantum computing is one of many things we're doing to imagine and prepare for what might be around the corner so that we can disrupt ourselves as opposed having others disrupt us" [66, 67]. Quantum computing promises to lead to discoveries and answer problems that scientists and businesses were unable to address.

Adversarial Strategy: The last strategy we identified was applications of quantum computing in situations where instantaneous responses are in order and where firms anticipate gaining advantages - or simply being able to compete at all in the future - from quantum computing. These applications involve "arms race" dynamics where firms anticipate facing other organizations with quantum computing capabilities. Adversarial applications include things like cybersecurity and financial trading. Take, for instance, applications in the financial sector where financial models often use many computationally intensive variables such as interest-rate yield curves, market assumptions, options thresholds, and various transaction costs. It is not unreasonable to expect that quantum computing will play a significant role in optimal trading trajectory, optimizing arbitrage opportunities, and optimal credit scoring [68]. Commerzbank experimented with the technology to optimize the loan portfolio selection process for vehicle leasing contracts and demonstrated more efficient methods $[69,70]$. Over the past few years, Fidelity has partnered with Amazon Bracket to develop a hybrid quantum algorithm that evaluates a potential list of stocks to determine which mix meets a predetermined target attribute index [71]. During the same period, Barclays and IBM partnered to research quantum methods and algorithms used for transaction settlement in capital markets to settle tens of thousands of trades, which conventional computers can only handle hundreds of trades [72]. Financial models often use many variables such as interest-rate yield curves, market assumptions, options thresholds, and various transaction costs. It is not unreasonable to expect that quantum computing will play a significant role in improving trading trajectories, optimizing arbitrage opportunities, and more precise credit scoring [73]. However, the capabilities for quantum computing in finance extend beyond traditional optimization problems. Monte Carlo machine learning algorithms align well for quantum deep learning, and firms have begun to run partial simulations to see if they beat conventional computing [52]. Goldman Sachs recently stated that quantum computing is five years away from beating traditional computers and using practical problems [74]. The firm teamed up with QC Ware to build a partial Monte Carlo simulation that uses the technology's current capabilities to produce a tenfold performance increase [74, 75]. William Zeng, head of quantum research at Goldman, has also stated the company is looking to build out derivative pricing or risk calculation algorithms, although no public announcement of them achieving a viable algorithm has been made [73]. The financial industry is one of the most competitive business areas, and quantum computers may provide firms the slight edge to beat out their competitors. These companies own significant amounts of cash and have shown a 
demonstrated willingness to explore quantum computers, so it should not be surprising that when quantum does become commercially viable, financial firms will be among the first to take advantage of its capabilities.

The last, potentially most impactful, and distant use of quantum computers is using the technology for factorization. In 1994, Peter Shor demonstrated that quantum computers could factor large numbers, which, if executed, can use cryptosystems public key to identify the private key [76, 77]. The U.S. National Institute of Standards and Technology (NIST) estimates that within 20 years, quantum computers will be able to break all public-key schemes, which poses risks to both national security and information security at business [9, 32]. Similarly, the U.S. National Academies of Sciences gathered leading academics and practitioners who concluded that the technology did not threaten public-key cryptosystems in the next decade [66]. Yet, the threat of quantum computers breaking encryption also comes with another promise: quantum encryption, making future communications more secure than they are today [43]. The U.S. and Chinese governments, AT\&T, Alibaba, Raytheon, and others, are exploring using entanglement to secure communications across locations [7]. Organizations do not yet need to worry about quantum computers breaking their secure communications; however, they must start preparing for the future. Every hack into a company's secure database leaves open the possibility that the data could be saved for future dates when technology catches up and can break encryption.

After reviewing the various strategies, we noticed that the discourse among the stakeholders in the different strategies diverged. To gain a qualitative perspective, we did a word frequency count of content across the four strategies. The conventional strategies were focused on specific tasks that improve domains such as energy and transportation. Terms such as "routing," “congestion," "time," "reduced," and "improvement" frequently appear in articles and white papers that focus on applications in this strategy basket. For options strategies, the discourse seems to identify techniques that quantum computing will reach "quantum supremacy" over conventional computers. Words of note include "optimizing," "cryptography," "neural networks," and "machine learning." Meanwhile, in the discovery strategy, the discussion focuses on the problems that the technology can address. Words of note include "chemistry," "energy," "discovery," "batteries," and "materials." The last strategy, adversarial, is concentrated in the areas and methods that the technology can soon improve, including "Monte Carlo," "portfolio," "risk," "securities," and "settlement." The development of quantum computing for business has created various strategies that each take different approaches to prepare their organizations for the future of computing.

\section{Discussion and Conclusion}

The excitement surrounding quantum computing rests in the expectation that the technology will solve computationally complex problems faster and answer previously deemed unsolvable due to the limits of technology. Already, quantum computers can take some problems and solve them orders of magnitude faster. In one widely discussed example, a quantum computer solved a problem in three minutes that engineers claimed would take a supercomputer thousands of years to solve [6]. The challenge facing quantum applications lies within the various layers of the quantum computing business ecosystem, which require hardware, infrastructure, software programs, and accessibility to reach commercially viable levels. Today's quantum computers can outperform conventional computers for some specific tasks; however, the vast majority of problems are still more easily solved by traditional computers [62]. As noted, problems well suited for quantum include quantum simulation, optimization, deep learning, and factorization [52], but many other applications are likely to emerge over time. Many firms, including Ford, Goldman Sachs, and the Cleveland Clinic, are experimenting with the Quantum Business Ecosystem to build capabilities in preparation for the day quantum hardware becomes commercially viable.

The fact remains that optimization problems can be some of the most computationally intensive applications of computing. The promise of quantum to hold many values at once leads to much excitement. As the NISQ era continues to develop in the next few years, applications will use hybrid quantum-classical algorithms to explore potential use cases. The impact of quantum computers on information is a long way away, but depending on the steps organizations take in the coming years, it may be the most impactful. In this work, we have (1) characterized the quantum computing business ecosystem and (2) identified four general contemporary quantum computing strategies. Future research should stay abreast of advancements as they arise and elaborate on these ideas and address their antecedents and consequences.

\section{References}

1. Moskvitch, K.: How AI and Quantum Could Help Fight Climate Change, https://ibm-

research.medium.com/earth-day-how-ai-and-quantumcould-help-fight-climate-change-4156fe6ee16d 
2. Moskvitch, K.: Quantum Computing and AI to Enable Our Sustainable Future, https://ibm-

research.medium.com/quantum-computing-and-ai-toenable-our-sustainable-future-58aa494cd4bc

3. Moskvitch, K.: Quantum \& AI: Accelerating Discovery to help the world Cope, https://ibmresearch.medium.com/quantum-ai-acceleratingdiscovery-to-help-the-world-cope-9ce61ce55c8c

4. Moskvitch, K.: Quantum Computing gains a first foothold in investment banking, https://ibmresearch.medium.com/quantum-computing-gains-a-firstfoothold-in-investment-banking-2806b280b8f

5. Press Release: New Study Reveals $81 \%$ of Fortune 1000 Decision-Makers Have a Quantum Computing UseCase In Mind For The Next Three Years, (2020)

6. Biamonte, J., Wittek, P., Pancotti, N., Rebentrost, P., Wiebe, N., Lloyd, S.: Quantum machine learning. Nature. 549, 195-202 (2017)

7. Cusumano, M.A.: The business of quantum computing. Commun. ACM. 61, 20-22 (2018)

8. MacQuarrie, E.R., Simon, C., Simmons, S., Maine, E.: The emerging commercial landscape of quantum computing. Nat. Rev. Phys. 2, 596-598 (2020)

9. Green, D., Soller, H., Oreg, Y., Galitski, V.: How to profit from quantum technology without building quantum computers. Nat. Rev. Phys. 1-3 (2021)

10. Trabesinger, A.: Quantum computing: towards reality. Nature. 543, S1-S1 (2017)

11. Jones, N.: Computing: The quantum company. Nat. News. 498, 286 (2013)

12. Preskill, J.: Quantum Computing in the NISQ era and beyond. Quantum. 2, 79 (2018)

13. Steane, A.: Quantum computing. Rep. Prog. Phys. 61, 117 (1998)

14. Matthews, D.: How to get started in quantum computing. Nature. 591, 166-167 (2021)

15. Hassija, V., Chamola, V., Goyal, A., Kanhere, S.S., Guizani, N.: Forthcoming applications of quantum computing: peeking into the future. IET Quantum Commun. 1, 35-41 (2020)

16. Mavroeidis, V., Vishi, K., Zych, M.D., Jøsang, A.: The impact of quantum computing on present cryptography. ArXiv Prepr. ArXiv180400200. (2018)

17. Preskill, J.: Quantum computing and the entanglement frontier. ArXiv Prepr. ArXiv12035813. (2012)

18. Pranav, G.: The Post-Quantum World: Quantum Computing Use Cases, https://www.protiviti.com/USen/insights/podcast-quantum-computing-use-cases

19. Gil, D., Mantas, J., Sutor, R., Kesterson-Townes, L., Flöther, F., Schnabel, C.: Coming soon to your business - Quantum computing. IBM Research Insights (2018)

20. Menard, A., Ostojic, I., Patel, M., Volz, D.: A game plan for quantum computing. McKinsey Q. (2020)

21. Mohseni, M., Read, P., Neven, H., Boixo, S., Denchev, V., Babbush, R., Fowler, A., Smelyanskiy, V., Martinis, J.: Commercialize quantum technologies in five years. Nat. News. 543, 171-174 (2017)

22. Rodriguez, J.: Some things I've learned about the quantum computing market,

https://jrodthoughts.medium.com/some-things-ive- learned-about-the-quantum-computing-market$2 \mathrm{e} 3 \mathrm{a} 91 \mathrm{c} 44 \mathrm{ddb}$

23. Buluta, I., Nori, F.: Quantum simulators. Science. 326, 108-111 (2009)

24. Bryant, D.: Architecting molecules that redefine luminescence, https://www.ibm.com/case-studies/jsrmitsubishi-keio/

25. Buchholz, S., Mariani, J., Routh, A., Keyal, A., Kishnani, P.: The realist's guide to quantum technolohgy and national security. The Deloitte Center for Government Insights (2020)

26. Byrum, J.: Quantum optimization: The future of operations research,

https://pubsonline.informs.org/do/10.1287/LYTX.2021.0 4.05/full/

27. Aaronson, S.: Read the fine print. Nat. Phys. 11, 291293 (2015)

28. Preskill, J.: Why I Coined the Term "Quantum Supremacy"

29. Brooks, M.: BEFORE THE QUANTUM

REVOLUTION. Nature. 574, 19-21 (2019)

30. Dietz, M., Henke, N., Moon, J., Backes, J., Pautasso, L., Sadeque, Z.: How quantum computing could change financial services. McKinsey \& Company (2020)

31. Carrel-Billiard, M., Garrison, D., Dukatz, C.: Think Beyond Ones and Zeros. Accenture Labs (2017)

32. Haddad, M., Schinasi-Halet, G., El Moutaouakil, A., Belhouchat, S., Jasmine, S.: Quantum Computing: A technology of the future already present. PwC (2019)

33. Gambetta, J.: IBM's Roadmap For Scaling Quantum

Technology, https://www.ibm.com/blogs/research/2020/09/ibmquantum-roadmap/

34. bp joins the IBM Quantum Network to advance use of quantum computing in energy, (2021)

35. Adachi, S.H., Henderson, M.P.: Application of quantum annealing to training of deep neural networks. ArXiv Prepr. ArXiv151006356. (2015)

36. Castelvecchi, D.: IBM's quantum cloud computer goes commercial. Nat. News. 543, 159 (2017)

37. Langione, M., Kumar, A., Tillemann-Dick, C., others: Where will quantum computers create value - and when. Boston Consulting Group (2019)

38. Russo, M., Thaker, A., Adam, S.: The coming quantum leap in computing. Boston Consulting Group (2018)

39. Piattini, M., Peterssen, G., Pérez-Castillo, R.: Quantum Computing: A New Software Engineering Golden Age. ACM SIGSOFT Softw. Eng. Notes. 45, 12 14 (2020)

40. Heim, B., Soeken, M., Marshall, S., Granade, C., Roetteler, M., Geller, A., Troyer, M., Svore, K.: Quantum programming languages. Nat. Rev. Phys. 1-14 (2020)

41. ExxonMobil strives to solve complex energy challenges, https://www.ibm.com/casestudies/exxonmobil/

42. Fretty, P.: Could Quantum Computing Solve Maritime Complexities?,

https://www.industryweek.com/technology-andiiot/article/21159784/could-quantum-computing-solvemaritime-complexities 
43. Harwood, S., Gambella, C., Trenev, D., Simonetto, A.: ExxonMobil \& IBM Explore Quantum Algorithms to Solve Routing Formulations, https://ibmresearch.medium.com/exxonmobil-ibm-scientistsexplore-state-of-art-quantum-algorithms-to-solverouting-formulations-e7ce39f8741c

44. Leprince-Ringuet, D.: IBM and ExxonMobil Are Building Quantum Algorithms to Solve Maritime Routing Problems, https://cacm.acm.org/news/250641ibm-and-exxonmobil-are-building-quantum-algorithmsto-solve-maritime-routing-problems/fulltext

45. Castellanos, S.: Volkswagen to Test Quantum Navigation App in Real Traffic, https://www.wsj.com/articles/volkswagen-to-testquantum-navigation-app-in-real-traffic-

11572553300\#: :text=The\%20app\%20uses\%20cloud\%2 Dbased,Hofmann\%2C\%20Volkswagen's\%20chief\%20in formation\%20officer., (2019)

46. Castellanos, S.: Amazon Rolls Out QuantumComputing Service; Select customers will be able to test quantum algorithms, hardware, (2019)

47. Krok, A.: VW wants to use quantum computing for traffic management, (2018)

48. Volkswagen ensures intelligent traffic management with quantum computers, (2018)

49. Washington, K.: Mass Navigation: How Ford Is Exploring the Quantum World with Microsoft to Help Reduce Congestion, https://medium.com/@ ford/massnavigation-how-ford-is-exploring-the-quantum-worldwith-microsoft-to-help-reduce-congestion-a9de6db32338

50. Lackey, B., Troyer, M.: Quantum impact: Optimization Solutions. Microsoft (2020)

51. IBM Announces Advances to IBM Quantum Systems \& Ecosystems, (2017)

52. Anderson, M.: Machine learning and quantum computing become BFFs [News]. IEEE Spectr. 54, 1414 (2017)

53. Fortson, D.: How RBS made a quantum leap into the future, (2020)

54. Pandya, R.: Detecting Brain Tumours using QNNs, https://medium.com/geekculture/detecting-braintumours-using-qnns-48f0e385db0, (2021)

55. Siemens Healthineers: Pioneering Healthcare with Quantum Computing, https://www.quantumtechcongress.com/blog/siemenshealthineers-pioneering-healthcare-with-quantumcomputing

56. Castellanos, S.: Financial Firms Wary Of Quantum Hackers, (2020)

57. IBM Developing New Cloud Services and Technology to Help Keep Data Secured from Future Fault-Tolerant Quantum Computers, (2019)

58. IBM Cloud Delivers Quantum-Safe Cryptography and Hyper Protect Crypto Services to Help Protect Data in the Hybrid Era, (2020)

59. Cyber Daily: Red Tape Frustrates Efforts to Protect Cities From Hackers | Financial Firms Prep for QuantumFueled Attacks, (2020)

60. Gopie, M.: Volkswagen developing quantum computing for battery simulation at molecular level, (2018)
61. Envisioning a new wave in power, https://www.ibm.com/case-studies/daimler/

62. Blum, J.: IBM, Exxon Mobil make quantum computing leap, https://www.chron.com/business/texasinc/article/IBM-Exxon-Mobil-make-quantum-computingleap-13526569.php

63. ExxonMobil and IBM to Advance Energy Sector Application of Quantum Computing, https://www.businesswire.com/news/home/20190107006 017/en/ExxonMobil-and-IBM-to-Advance-EnergySector-Application-of-Quantum-Computing

64. D-Wave Announces General Availability of First Quantum Computer Built for Business, (2020)

65. Cleveland Clinic and IBM Unveil Landmark 10-Year Partnership to Accelerate Discovery in Healthcare and Life Sciences, (2021)

66. Harris, M.: Ford Signs Up to Use NASA's Quantum Computers, (2018)

67. Ford, NASA to Work Together to Apply Quantum Computing in Autonomous Vehicle Research Programs, (2018)

68. Orus, R., Mugel, S., Lizaso, E.: Quantum computing for finance: Overview and prospects. Rev. Phys. 4, 100028 (2019)

69. Fujitsu Quantum-Inspired Digital Annealer Delivers Securitisation Optimisation for main incubator, (2019)

70. Fujitsu collaborates with Commerzbank on securitisation project, (2019)

71. Schouela, A.: Exploring Quantum Computing with Amazon Web Services. Fidelity, Boston, MA (2020)

72. Saran, C.: Barclays demonstrates proof-of-concept quantum clearing algorithm,

https://www.computerweekly.com/news/252472462/Barc lays-demonstrates-proof-of-concept-quantum-clearingalgorithm

73. Crosman, P.: Goldman Sachs moves a step closer to quantum computing, (2021)

74. Waters, R.: Quantum computing just 5 years away, says Goldman, (2021)

75. Goldman Sachs, QC Ware Design Quantum Algorithms, https://www.marketsmedia.com/goldmansachs-and-qc-ware-design-quantum-algorithms/

76. Shor, P.W.: Algorithms for quantum computation: discrete logarithms and factoring. In: Proceedings 35th annual symposium on foundations of computer science. pp. 124-134. Ieee (1994)

77. Shor, P.W.: Polynomial-time algorithms for prime factorization and discrete logarithms on a quantum computer. SIAM Rev. 41, 303-332 (1999) 